\title{
Factors Affect the Survival Status of HIV Infected Patients under ART: The Case of Wolisso Catholic Hospital, Wolisso, Ethiopia, 2016.
}

Alemayehu Siffir Argawu ( $\sim$ alex089973@gmail.com )

Ambo University https://orcid.org/0000-0002-4444-0513

\section{Research Article}

Keywords: HIV Patients, Survival Analysis, Hazard Rate, Risk factors

Posted Date: December 23rd, 2020

DOl: https://doi.org/10.21203/rs.3.rs-133139/v1

License: (1) (1) This work is licensed under a Creative Commons Attribution 4.0 International License.

Read Full License 
Factors Affect the Survival Status of HIV Infected Patients under ART: The Case of Wolisso Catholic Hospital, Wolisso, Ethiopia, 2016

Principal Investigator: Alemayehu Siffir Argawu alex089973@gmail.com

Co-investigator: Bedane Gemechu bedane2020@gmail.com

Department of Statistics, CNCS, Ambo University, Ethiopia

\begin{abstract}
INTRODUCTION: Monitoring the length of survival after diagnosis is, therefore, an important component of the surveillance of AIDS. It provides a basis for evaluating individual prognostic factors. The survival of patients with AIDS may depend on a variety of factors that are socio-demographic and clinical factors. The study is designed to identify factors that affect the survival status of HIV infected patients under ART in Wolisso, Ethiopia.

METHODS: This is a retrospective study based on cases of HIV infected patients of age 15 years and above who have started ART from September 2010 to August 2013 and followed until February 30th, 2014. To analyse the data descriptive statistics, univariate and multivariate analyses were used. The descriptive analysis indicates that out of the total of 658 $H I V$-infected patients, 533(81\%) are censored and 125(19\%) are uncensored in the study. And, from 125 uncensored patients, 60(9.1\%) patients have died and 65(9.9\%) are dropped out cases. The overall mean estimated survival time of patients under the study was 33.76(95\% CI: 32.6-34.9) months.
\end{abstract}

RESULTS: The Kaplan Meier method was used to estimate the survival time and Cox's regression model was employed to identify the covariates that have a statistically significant effect on the survival of HIV-infected patients. Based on the log-rank test, there was a significant difference in survival experience between the various categories of gender, marital status, work type, duration of pre-ART, regimen type, functional status, TB type, WHO clinical stage, condom use, and substance abuse (like smoking or alcoholic). And, the multivariate analysis of the Cox regression model gives that CD4 count, functional status, TB type, WHO clinical stage, condom use, and substance abuse are significant risk factors for survival of HIV-infected patients.

CONCLUSION: The patients with low CD4 count, WHO stage III or IV, being ambulatory or bedridden, extra-pulmonary, not used condom, and substance abuse were associated with a high risk of death or dropped out the ART of the patients. Therefore, it is recommended that these at-risk patients need careful monitoring to improve their survival times by the concerned bodies.

Keywords: HIV Patients, Survival Analysis, Hazard Rate, Risk factors 


\section{INTRODUCTION}

\section{Back ground of the study}

Studying the history of disease progression due to HIV/AIDS and the treatments are useful for the purpose of developing treatment guidelines, modelling the epidemic and prioritizing and allocating resources. Health care planning depends on good knowledge of prevalence, which requires an accurate understanding of survival patterns. Monitoring the length of survival after diagnosis is, therefore, an important component of the surveillance of AIDS. It provides a basis for evaluating individual prognostic factors. In addition, differences in survival may also reflect differences in access to health care (e.g. access to testing, counselling, preventive treatment). The survival of patients with AIDS may depend on a variety of factors including host factors, the patterns of diseases present, access to health care, diagnostic routines and therapeutic interventions (Robert et al., 1995).

Long term sustainable treatment is one choice for people living with HIV. Not only can medications slow the progression of the infection, but can also markedly suppress the virus, thereby restoring the body's immune function and permitting many HIV-infected individuals to lead a normal life. People living with HIV take a number of medications like cotrimoxazole, fluconazole, and other medications depending on the type of the opportunistic illness they have. Tuberculosis is the most common opportunistic infection complicating HIV infection especially in developing countries, and may occur at any stage in the course of immunodeficiency. TB/HIV co-infection significantly changes the natural history of both diseases and it is recognized as a major setback of both HIV and tuberculosis infection control program (Egger et al., 2002 and Palella et al., 2003).

Development of highly active antiretroviral treatment (ART) in the mid-1990s revolutionized the care of HIV-infected patients and led to marked reductions in HIV-associated morbidity and mortality in many industrialized countries. ART has clearly shown to be effective in reducing mortality among those who remain in treatment and adhere to therapy (Egger et al., 2002 and Lawn et al., 2008). In recent years in developing countries with a high burden of AIDS, ART has become more widely available. According to estimation by the World Health Organization (WHO, 2010), about 6, 650000 patients were receiving ART in low- and middle income countries by the end of 2010 (UNAIDS), this is a huge improvement from the levels in 2003 (UNAIDS et al., 2011 and WHO, 2010). From those studies, the two subSaharan African countries, Botswana and Rwanda, have achieved universal access target (treatment coverage of $80 \%$ or more of patients in need) at the end of 2009 while countries such as Ethiopia, Zambia, Namibia and Senegal are moving closer to the same target having covered $50-80 \%$ of patients in need of treatment. Due to this morbidity and mortality among HIV-infected persons have dramatically decreased (Mocroft et al., 1998 and CDCP, 2001).

The primary goals of antiretroviral therapy are preventing HIV-related morbidity and mortality, and improving quality of life by restoring immunologic function through suppression of viral load (Hoffmann et al., 2007). Many studies have reported high early mortality among patients starting antiretroviral treatment in sub-Saharan Africa (Lawn et al., 2008). One of the reasons cited for this is that despite better availability of ART, people are 
still diagnosed late and thus start ART at later stages of the HIV disease (Braitstein et al., 2006).

In Ethiopia, there were more than 222,000 patients on antiretroviral treatment at the end of 2010 (UNAIDS, 2011, and WHO, 2010). ART has improved survival of patients with HIV/AIDS and improved the quality of life of patients in the country (Jerene et al., 2006). And, study by (Sibhatu et al., 2011) examined the mortality and its predictors among a cohort of HIV infected patients on antiretroviral treatment retrospectively followed for three years. In Ethiopia, there are no more studies reporting the long term survival of patients on antiretroviral treatment. But, such studies could provide valuable information to evaluate the ART program in the country.

\section{Statement of the problem}

It is recommended that HIV infected patients should start to take ART in order to reduce AIDS related mortality and morbidity, or to improve their quality of life. Moreover, death and dropout during ART and shortened life are recognized in HIV patients. There is a misunderstanding among the patients who do not follow up ART on time be experiencing decreased quality of life and survivalist. The incomplete or dropout of ART in the patients causes fast death as compared to those who follow up ART properly. And, it can be estimated the death and dropout rates of the patients in the hospital, since there is no studies conducted in this hospital. Moreover, the research problems include: assessing and measuring survival/death status; determining the risk factors of survival/death of HIV patients; looking at implications of observing significant risk factors and evaluating the impacts of influential or risk factors on survival/death.

\section{Objective of the Study}

The general objective of the study is to identify factors that affect the survival of HIV infected patients on ART in SLCHCN.

\section{The specific objectives are;}

* To estimate the survival times of HIV infected patients on ART.

* To determine the hazard rate of the survival of the patients.

* To estimate the death and dropout rates of the patients.

\section{Significance of the study}

The outcome of this study will provide information about the risk factors or the most influential covariate that have significant impact on survival of HIV patients during following of ART. The beneficiaries from this study are SLCHCN to give awareness for HIV infected patients in the risk factors of the survival times of patients on ART and the whole societies, all HIV infected patients to follow ART on time, Ambo University as a research finding and others concerned body such as Saint Luke Catholic Hospital and College of Nursing and Ministry of health. 


\section{MATERIALS AND METHODOLOGY}

\section{Study area and data type}

The data in the study was obtained from Saint Luke Catholic Hospital and College of Nursing (SLCHCN) at Wolisso town, south western Oromia regional state of Ethiopia. It is 66 kilo meter far from Ambo town.

The study is a retrospective study type. The resulting data set comprises all cases of HIV infected patients who have started ART between September 2010 to August 2013 and followed for the outcomes of either the event (death or drop out) or censored (transferred out to other hospital/health centres or on follow up at the end of study time). The end of the follow up time was February $30^{\text {th }}, 2014$.

\section{Sample size determination}

A total of 674 patients were treated with ART in the hospital SLCHNC during the study period from September 2010 to August 2013. And, all those patients are older than 15 year. However, the study included $658 \mathrm{HIV}$ infected patients under ART for whom the data/variables of interest are completed. This number is not too large then we take all patients as a total sample size to maximize the sample size which makes the research quality high. Further analysis was carried out using SPSS 20 soft ware.

\section{The response variable}

The response (dependant) variable is the survival time of HIV patients, the length of time from ART start date until the date of event or censor measured in months. In this study, the event is death or drop out from ART.

\section{Predictor variables}

There are 18 covariates or predicators are detailed below in the study.

Sex (male, female), age $(15-24,25-34,35-44,45-54, \geq 55$ years), marital status (never married, married, other), religion (orthodox, muslin, protestant), education level (no education, primary, secondary and above), work type (farmer, merchant, employer, house wife, daily labour and other), have children (yes, no), house hold size (one, two, three, above three), baseline weight (kg), TB co-infection (not co-infected, pulmonary, extra- pulmonary), baseline cd4 cell count (cells $/ \mathrm{mm}^{3}$ ), WHO clinical stage (stage I, stage II, stage III, stage IV), functional status (working, ambulatory, bedridden), duration for pre art ( $\leq 6$ months, $7-12$ months, $>12$ months), ART regimen type (AZT based, D4T based, TDF based), awareness of ART (poor $(-)$, good $(+)$, very good $(++)$, excellent $(+++)$ ), substance abuse like smoking or alcohol (yes, no) and condom usage (never, sometimes, always).

\section{Kaplan Meier survival function}

Kaplan Meier survival analysis is a descriptive procedure for the time to event variables (censored or non censored data) in cases where time is the most prominent variable.

Generally, the survival of the patient who had started to follow the recommended treatment (like ART) of HIV patients is formulated as:

$\widehat{S}(t)=\prod_{j=1}^{k}\left(1-\frac{d_{j}}{n_{j}}\right)$ 
Where $t \in[t(j), t(j+1)], t(j)$ is the $j^{\text {th }}$ distinct event time, $j=1,2, \ldots, k, k$ is the last event time, $d_{j}$ is the number of individuals who experience the event or failure at time $t(j)$, and $n_{j}$ is the number of individuals who have not yet experienced the event at that time and are therefore still at risk of experiencing it.

\section{Comparison of survival curves}

The log rank test statistic for comparing two groups is given by:

$Q=\frac{\left[\sum_{j=1}^{m}\left(d_{1 j}-\hat{e}_{1 j}\right)\right]^{2}}{\sum_{j=1}^{m} \hat{v}_{1 j}}$

Where $m$ is the number of rank ordered event times, $d_{1 j}$ is the observed number of events in group 1 at event time $t_{j}, \hat{e}_{1 j}=\frac{n_{1 j} d_{j}}{n_{j}}$ is the expected number of events corresponding to $d_{1 j}$, $\hat{v}_{1 j}=\frac{n_{1 j} n_{0 j} d_{j}\left(n_{j}-d_{j}\right)}{n_{j}^{2}\left(n_{j}-1\right)}$ is the variance of the number of events $d_{1 i}$ at time $t_{j}, n_{1 j}$ is the number of individuals at risks in group 1 just prior to event time $t_{j}, n_{j}$ is the number of individuals at risk in both groups 1 and 2 just prior to event time $t_{j}$, and $d_{j}$ is the total number of events in both groups at time $t_{j}$.

\section{The Cox proportional hazard model}

Cox proportional hazards regression model is a useful technique for assessing the relationship between survival times and a set of explanatory variables, (Cox, 1972). The model assumes that the underlying hazard rate (rather than survival time) is a function of the independent variables (covariates).

The model can be written as:

$h(t, X, \beta)=h_{0}(t) e^{\beta^{\prime} X}$

Where $h(t, X, \beta)$ denotes the resultant hazard given that the values of the covariates for the respective case $X=\left(x_{1}, x_{2}, \ldots, x_{m}\right)$ and the respective survival time $t$, the term $h_{0}(t)$ is called the baseline hazard; it is the hazard for the respective individual when all independent variable values are equal to zero, and $\beta=\left(\beta_{1}, \beta_{2}, \ldots, \beta_{m}\right)$ : is a column vector of $\mathrm{m}$ regression parameters.

Hazard Rate (HR) is defined as the hazard for one individual divided by the hazard for a different individual. Then, the mathematical expression of the HR equation is:

$$
H R=\frac{h\left(t, x^{*}, \beta\right)}{h(t, x, \beta)}=\frac{\exp \left(\beta^{\prime} x^{*}\right)}{\exp \left(\beta^{\prime} x\right)}=\exp \left[\beta^{\prime}\left(x^{*}-x\right)\right]=\text { constant }
$$




\section{RESULT AND INTERPRETATION}

\section{Baseline characteristics of the study}

From the total of $658 \mathrm{HIV}$ infected patients, 417 (63.3\%) were females, 396 (60.2\%) were married, $308(46.8 \%)$ had a primary school education, 467(71\%) were orthodox, only 62(9.4\%) were employers, 508(77.2\%) had children, 338(51.4\%) had more than three members in the household, 453(68.8\%) were not experienced to substance abuse, $310(47.1 \%)$ had never used condom, 446(67.8\%) were working functional status and only $167(25.4 \%)$ had no awareness of ART, and 310(47.1\%) aged from 25 to 34 years with the mean and median are 32.8 years and 30 years, respectively. From the clinical characteristics of the patients, the regimen TDF based was frequently prescribed for 506(76.9\%) patients and $241(36.6 \%)$ were on the worst stage as III or IV. And, from $129(19.6 \%) \mathrm{HIV} / \mathrm{TB}$ co-infected patients, $81(12.3 \%)$ and $48(7.3 \%)$ of them had been infected by pulmonary and extrapulmonary TB types, respectively. The mean CD4 count and weight of patients at start of ART are 202.2 cells $/ \mathrm{mm}^{3}$ (median is 181.5 cells $/ \mathrm{mm}^{3}$ ) and $52 \mathrm{~kg}$ (median is also $52 \mathrm{~kg}$ ), respectively.

\section{Descriptive survival analyses}

Among the total of $658 \mathrm{HIV}$ infected patients, 533(81\%) are censored and 125(19\%) are uncensored in the study. Out of 533 censored patients, 484(73.6\%) patients are follow up and 49(7.4\%) patients are transfer out cases. And, from 125 uncensored patients, 60(9.1\%) patients are died and 65(9.9\%) are dropped out cases.

The median survival time of the patients is 18 months. The minimum follow up time was one month and the maximum was forty one months. The overall mean estimated survival time of patients under the study was 33.76(95\% CI: 32.6-34.9) months. The graph of cumulative survival function is depicted in figure 1 below showing that the estimated cumulative survival probability of the patients is decreased from 1.00 to 0.78 with an increasing of ART time.

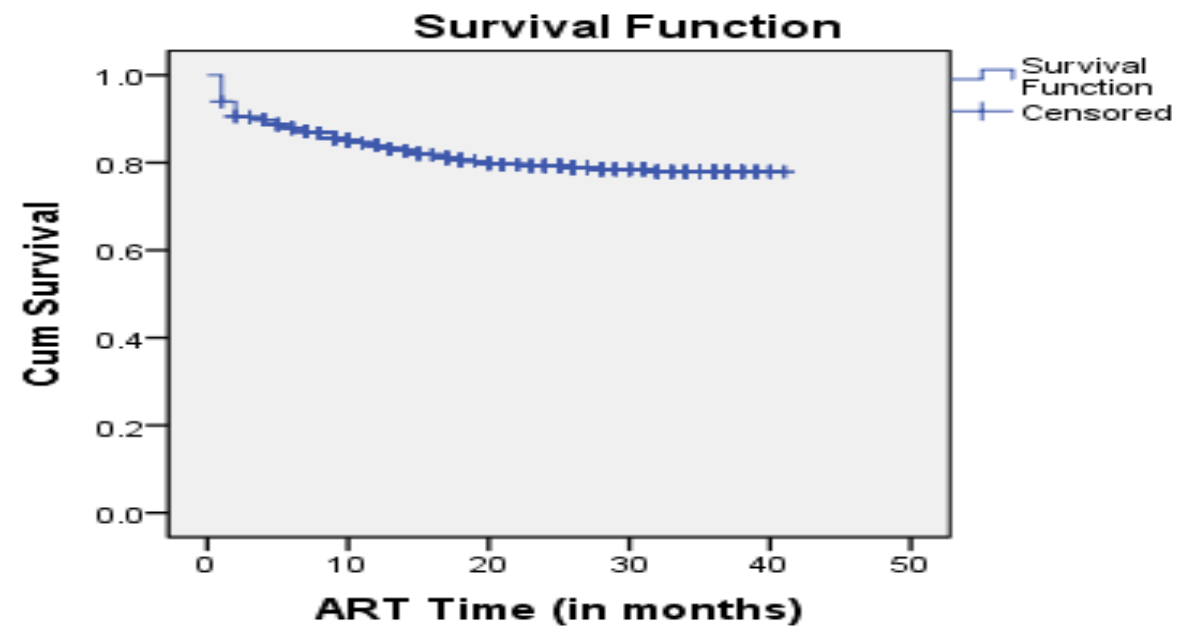

Figure 1: The plot of the overall estimate of Kaplan Meier cumulative survivor function of HIV patients under ART in SLCHCN, Wolisso, 2014. 


\section{Log rank test using Kaplan Meier estimates}

Based on the log-rank test, there was a significant difference in survival experience between the various categories of gender, marital status, work type, duration of pre-ART, regimen type, functional status, TB type, WHO clinical stage, condom use and substance abuse. Some of results are shown in table 1 and figures 2 and 3 below. And, a close examination from this table and figure reveal that patients who are female, married, working as house wife, followed pre-ART more than one year, having AZT ART type, working as functional status, no TB co-infection, WHO clinical stage I, used condom always and those who were not addicted by any substance had better survival times.

\section{Results of the Cox proportional hazards model}

Let us begin the variables that are found to be significantly associated with the survival of patients in the fitted Cox regression model are WHO stage, TB co-infection, functional status, condom use and substance use at 5\% level of significance in table 2 below.

The reference category for WHO clinical stage is patients who are in primitive clinical stage I. The hazard rate for clinical stage II patient (HR: 5.626, 95\% CI: 1.302- 24.304) is 5.6 times higher than that of clinical stage I patient. And, the hazard rate of the patients in clinical stage III (HR: 8.688, 95\% CI: 1.993- 37.878) is 8.6 times higher than that of clinical stage I patient. Similarly, the hazard rate of patients in advanced clinical stage IV (HR: 8.982 (95\% CI: $2.153-38.825$ ) is 8.9 times higher than that of clinical stage I patient.

TB site or location has a significant impact on survival/death or dropout status of patients. The hazard rate of patients with extra-pulmonary TB (HR: 2.039, 95\% CI: $1.254-3.316$ ) is 2.039 times higher than that of TB uninfected patients. The $95 \%$ confidence interval indicates that the hazard rate goes to a maximum of 3.316 and a minimum of 1.254.

The estimated hazard rates of a patient with ambulatory and bedridden functional status in comparison with working group are 3.520 (95\% CI: 1.985 - 6.243) and 6.220 (95\% CI: 3.385-11.430), respectively. This implies that the hazard rate of a patient in ambulatory and bedridden categories of functional status is, approximately, 3.5 times and 6 times higher than that of the working patients, respectively.

The baseline CD4 cell count has been found to have a significant impact on the death or dropout of HIV infected patients (HR: 0.998, 95\% CI: 0.996- 1.00). For appropriateness of interpretation we take the estimated hazard ratio for a 50 cells $/ \mathrm{mm}^{3}$ increase in the baseline CD4 cell count is $0.91=\exp (-0.002 * 50)$ showing that patients whose CD4 cell count is higher by 50 cells $/ \mathrm{mm}^{3}$ die or dropout at lower hazard rate of $9 \%$ controlling for other variables in the model.

The hazard rates for patients who used condom sometimes and always are $0.328(95 \% \mathrm{CI}$ : $0.188-0.572$ ) and 0.302 (95\% CI: 0.148- 0.617) in comparison with patients who never used condom, respectively. The result shows that the hazard rate among patients who used condom sometimes is 0.328 times lower than (decreased by $67.2 \%$ ) that of patients who never used condom. The hazard rate for patients who have not abused in substance (like alcohol or 
smoking) is 0.413 (95\% CI: $0.280-0.610)$ with that of patients who abused in substance. This implies that the hazard rate among patients who are not abused for alcohol or cigarette is 0.413 times lower than (decreased by $58.7 \%$ ) that of patients who are abused.

Table 1: The overall comparisons test of equality of survival distributions on HIV patients on ART using different covariates at 5\% level of significant (at SLCHCN, Wolisso, September 2010 to August 2013).

\begin{tabular}{|c|c|c|c|}
\hline Covariate/factor & $\begin{array}{l}\text { Mean Survival } \\
\text { time }\end{array}$ & Chi-square & $\begin{array}{l}\text { Log-rank } \\
\text { p value }\end{array}$ \\
\hline $\begin{array}{l}\text { Gender: (Male) } \\
\text { Female }\end{array}$ & $\begin{array}{l}32.15 \\
34.76\end{array}$ & 4.71 & 0.03 \\
\hline $\begin{array}{l}\text { Marital status: (Never married) } \\
\text { Married } \\
\text { Others }\end{array}$ & $\begin{array}{l}30.29 \\
34.96 \\
32.39 \\
\end{array}$ & 7.52 & 0.023 \\
\hline $\begin{array}{l}\text { Work type: (Farmer) } \\
\text { Merchant } \\
\text { Employer } \\
\text { House wife } \\
\text { Daily labor/other }\end{array}$ & $\begin{array}{l}31.34 \\
35.68 \\
34.91 \\
35.77 \\
30.24 \\
\end{array}$ & 12.81 & 0.012 \\
\hline $\begin{array}{l}\text { Substance use: (Yes) } \\
\text { No }\end{array}$ & $\begin{array}{l}25.78 \\
37.32 \\
\end{array}$ & 92.91 & 0.000 \\
\hline $\begin{array}{l}\text { Condom use: (Never) } \\
\text { Sometimes } \\
\text { Always }\end{array}$ & $\begin{array}{l}26.77 \\
38.23 \\
39.76 \\
\end{array}$ & 87.61 & 0.000 \\
\hline $\begin{array}{l}\text { Functional status: (Working) } \\
\text { Ambulatory } \\
\text { Bed ridden }\end{array}$ & $\begin{array}{l}39.33 \\
27.80 \\
13.41 \\
\end{array}$ & 319.17 & 0.000 \\
\hline $\begin{array}{l}\text { Duration for Pre-ART: }(<=6 \\
\text { months }) \\
7-12 \text { months } \\
>12 \text { months }\end{array}$ & $\begin{array}{l}31.53 \\
34.13 \\
37.19\end{array}$ & 24.76 & 0.000 \\
\hline $\begin{array}{l}\text { ART regimen: (AZT based) } \\
\text { D4T based } \\
\text { TDF based }\end{array}$ & $\begin{array}{l}33.99 \\
16.67 \\
33.74\end{array}$ & 11.01 & 0.004 \\
\hline $\begin{array}{l}\text { TB type: (Not co-infected) } \\
\text { Pulmonary } \\
\text { Extra-pulmonary }\end{array}$ & $\begin{array}{l}36.51 \\
29.18 \\
10.35 \\
\end{array}$ & 215.35 & 0.000 \\
\hline $\begin{array}{l}\text { WHO stage: (I) } \\
\text { II } \\
\text { III } \\
\text { IV }\end{array}$ & $\begin{array}{l}39.61 \\
36.85 \\
27.57 \\
17.13\end{array}$ & 163.76 & 0.000 \\
\hline
\end{tabular}

The first values (in bracket) are the reference categories of the variables. 
Table 2: Results of the multivariable proportional hazards Cox regression model containing the variables significant at 5\% level of HIV patients under ART in SLCHCN, Wolisso, 2014.

\begin{tabular}{|l|c|c|c|c|c|c|c|c|}
\hline Variables & $\hat{B}$ & SE & Wald & DF & Sig. & HR & \multicolumn{2}{|c|}{$95 \%$ CI for HR } \\
\cline { 7 - 10 } & & & & & & & Lower & Upper \\
\hline CD4 & -0.002 & 0.001 & 4.526 & 1 & 0.033 & 0.998 & 0.996 & 1.000 \\
\hline Functional Status & & & 34.693 & 2 & 0.000 & & & \\
(Working) & & & & & & & & \\
Ambulatory & 1.258 & 0.292 & 18.533 & 1 & 0.000 & 3.520 & 1.985 & 6.243 \\
Bedridden & 1.828 & 0.310 & 34.673 & 1 & 0.000 & 6.220 & 3.385 & 11.430 \\
\hline WHO Stage (I) & & & 9.680 & 3 & 0.002 & & & \\
II & 1.727 & 0.747 & 5.354 & 1 & 0.021 & 5.626 & 1.302 & 24.304 \\
III & 2.162 & 0.751 & 7.281 & 1 & 0.004 & 8.688 & 1.993 & 37.878 \\
IV & 2.188 & 0.776 & 7.588 & 1 & 0.006 & 8.982 & 2.153 & 38.825 \\
\hline TB Type & & & 11.934 & 2 & 0.003 & & & \\
(Not co-infected) & & & & & & & & \\
Pulmonary & -0.230 & 0.259 & 0.786 & 1 & 0.375 & 0.795 & 0.478 & 1.321 \\
Extra Pulmonary & 0.712 & 0.248 & 8.244 & 1 & 0.004 & 2.039 & 1.254 & 3.316 \\
\hline Condom Use (Never) & & & 22.420 & 2 & 0.000 & & & \\
Sometimes & -1.115 & 0.284 & 15.445 & 1 & 0.000 & 0.328 & 0.188 & 0.572 \\
Always & -1.198 & 0.365 & 10.788 & 1 & 0.001 & 0.302 & 0.148 & 0.617 \\
\hline Substance Use & -0.883 & 0.198 & 19.847 & 1 & 0.000 & 0.413 & 0.280 & 0.610 \\
\hline
\end{tabular}

The first values (in bracket) are the reference categories of the variables.

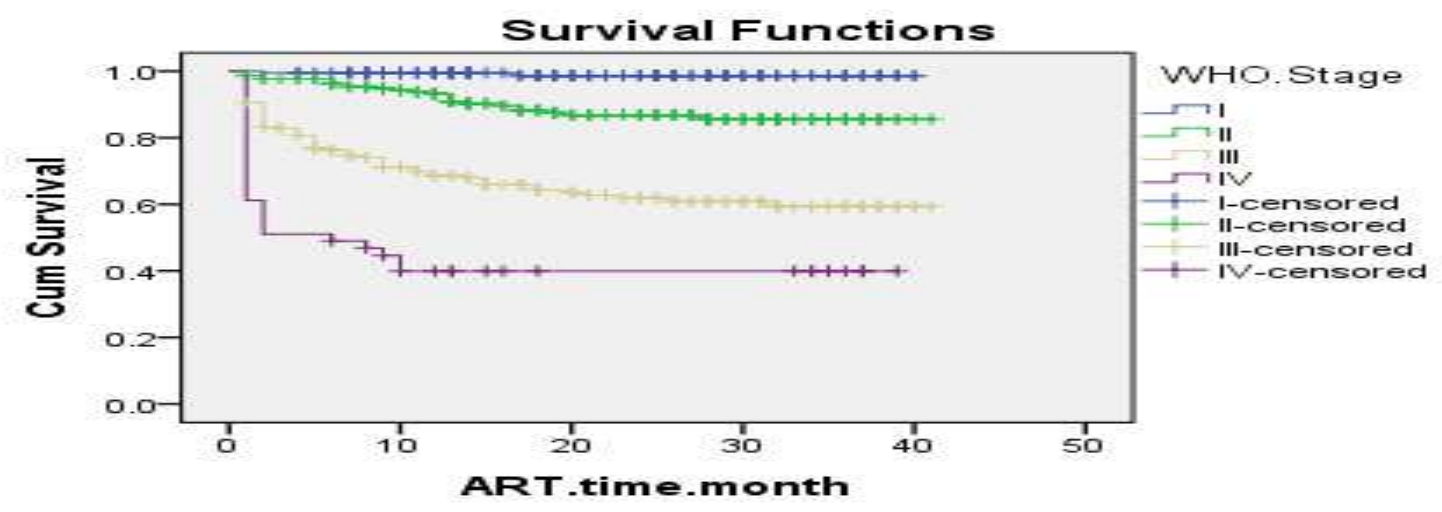

Figure 2: The plot of Kaplan Meier survival estimate for WHO clinical stage 


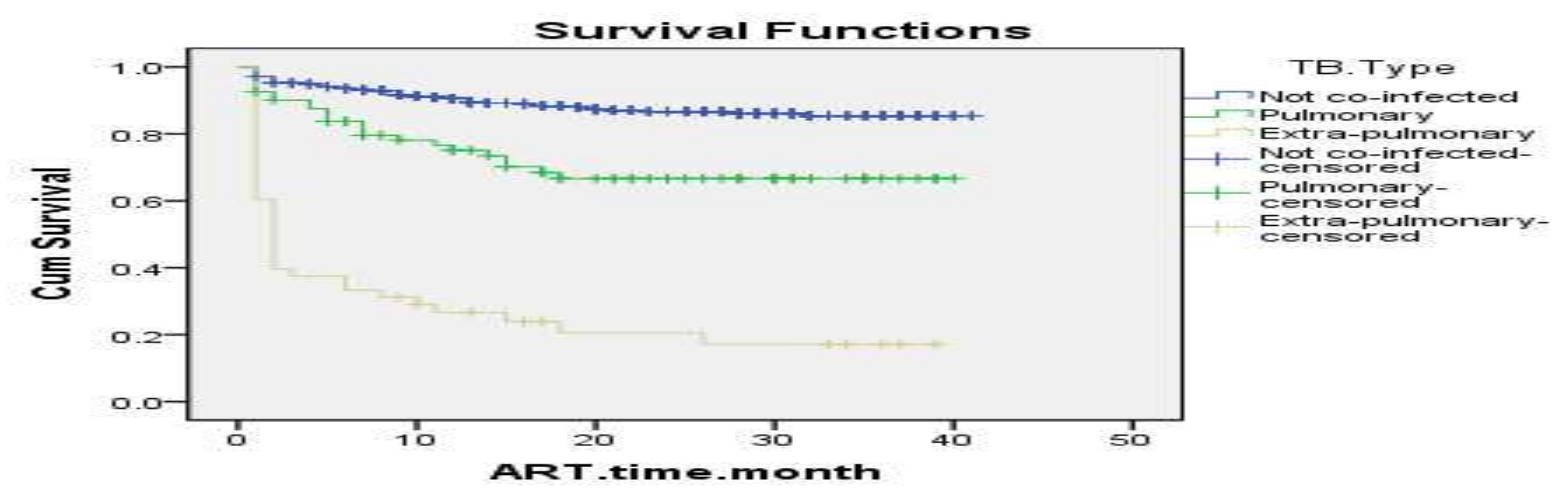

Figure 3: The plot of Kaplan Meier survival estimate for TB type

\section{Conclusions}

The study determined that factors that affect the survival status of HIV infected patients under ART in Saint Luke Catholic Hospital and College of Nursing (SLCHCN), Wolisso/Ethiopia, using the methods of survival analysis. The overall mean estimated survival time of patients under the study was 33.76(95\% CI: 32.6-34.9) months.

Based on the log-rank test, there was a significant difference in survival experience between the various categories of gender, marital status, work type, duration of pre-ART, regimen type, functional status, TB type, WHO clinical stage, condom use and substance abuse (like smoking or alcoholic). And, from the multivariate analysis of Cox regression model gives that CD4 count, functional status, TB type, WHO clinical stage, condom use and substance abuse are significant risk factors for survival of HIV infected patients.

Gender type, age interval, marital status, work type, having children, time duration for preART, regimen type and weight are significant factors in un-variable analysis but not in the multivariable analysis.

\section{Recommendations}

* Health workers and other ART clinic staffs should plan for more frequent contacts with patients during the early phase of treatment in order to prevent the many deaths or dropout that occur during the early weeks of ART. Moreover, the factors those are causes for or associated with increased rate of deaths or dropout in the early stages of ART initiation need to be explored.

* Having lower CD4 count, pulmonary or extra-pulmonary TB, being bedridden and WHO clinical stage III or IV are indicators of the progression of the disease. Therefore, patients should be informed about the need for early diagnosis of HIV infection and starting treatment early is very important.

* TB test must be done for people living with HIV in order to mitigate further deterioration of their health.

* HIV infected patients under ART should have awareness about the hazard of the risk factors identified in this study.

* Health workers and peer educators and data clerks, working with patients under ART, should be given special training to improve the quality of the data records of patients. 
Moreover, attempt should be made to investigate the causes of deaths that occurred out of hospitals, and mechanisms should be devised to trace patients lost to follow up (dropout).

* Furthermore the patients with low CD4 count, WHO stage III or IV, being ambulatory or bedridden, extra-pulmonary, not used condom and substance abuse are associated with high risk of death or dropped out the ART of the patients. Therefore, it is recommended that these at risk patients need a careful monitoring to improve their survival times.

\section{Acknowledgments}

We would like to extend our gratitude for Ambo University for its financial support and collaboration. Our thanks also went to St. Luke Catholic Hospital and College of Nursing and all ART clinic staffs for their cooperation during data collection.

\section{Competing interests}

The authors declare that there no competing interests.

\section{Authors' contributions}

Alemayehu Siffir Argawu developed the proposal, designed, involved in data collection, analyzed, writes up and drafted the manuscript. Bedane Gemechu was involved in editing and revising the research analysis. Both of the authors have read and approved the final version of the manuscript.

\section{References}

Braitstein P, Brinkhof MW, Dabis F, Schechter M, Sprinz E, Seyler C, et al: Mortality of HIV-1-infected patients in the first year of antiretroviral therapy: comparison between low-income and high-income countries. Lancet 2006, 367:817-824.

Cox, D.R, 1972. Regression models and life-tables. Journal of Reg Stat Soc 34: 187-220.

Egger M, May M, Chêne G, Phillips AN, Ledergerber B, Dabis F, Reiss P, et al: Prognosis of HIV-1-infected patients starting highly active antiretroviral therapy: $a$ collaborative analysis of prospective studies. Lancet 2002, 360:119-129.

Hoffmann C, JK R, Kamps S.: HIV Medicine 2007. Paris: Flying Publishers; 2007.

Jerene D, Naess A, Lindtjørn B: ART at a district hospital in Ethiopia prevents death and tuberculosis in a cohort of HIV patients. AIDS Res Ther 2006, 3:10.

Kaplan EL, Meier P. Nonparametric estimation from incomplete observations. Journal of the American Statistical Association 1958; 53: 457-481.

Lawn SD, Harries AD, Anglaret X, Myer L, Wood R: Early mortality among adults accessing ART programmes in sub-Saharan Africa. AIDS 2008, 22:1897-1908.

Mocroft A, Vella S, Benfield TL, Chiesi A, P, D'Arminio Monforte A, Yust I, Bruun JN, Phillips AN, Lundgren JD: Changing patterns of mortality across Europe in 
patients infected with HIV-1. Euro SIDA Study Group. Lancet 1998, 352:17251730.

Palella FJ, Deloria-Knoll M, Chmiel JS, Moorman AS, Wood KC, and Greenberg AE. HIV Outpatient Study Investigators: Survival benefit of initiating ART in HIV infected persons in different CD4+ cell Strata. Ann Intern Med 2003, 138:620-626.

Robert Z, Gilbert R, Jean P. Survival differences in Austrian patients with the Acquired Immunodeficiency Syndrome. European Journal of Epidemiology 1995; 11:519526.

Sibhatu B, Ayalu R and Tesfaye D, 2012. Predictors of mortality among HIV infected patients taking antiretroviral treatment in Ethiopia: a retrospective cohort study. Journal of Biomedical Central, 2012.

UNAIDS: Global report: UNAIDS report on the global AIDS epidemic 2010. vol. 10.11. Geneva: Joint United Nations Program me on HIV/AIDS (UNAIDS); 2010.

UNAIDS, WHO, UNICEF: Global HIV/AIDS response - Epidemic update and health sector progress toward universal access Progress report 2011. Geneva: UNAIDS; 2011.

WHO: Towards universal access scaling up priority HIV/AIDS interventions in the health sector Progress report 2010. Geneva: World Health Organization; 2010. 
Figures

Survival Function

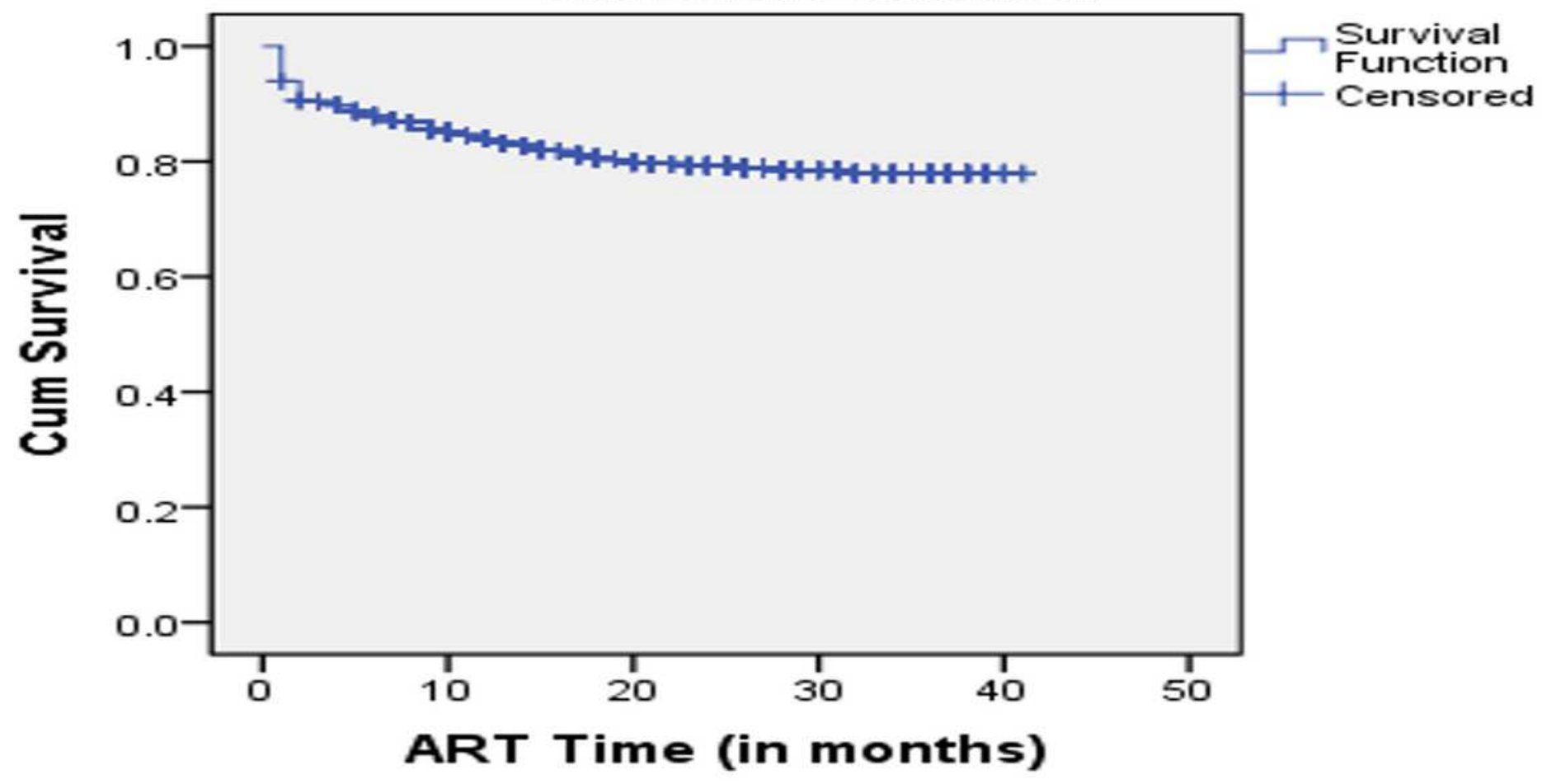

Figure 1

The plot of the overall estimate of Kaplan Meier cumulative survivor function of HIV patients under ART in SLCHCN, Wolisso, 2014.

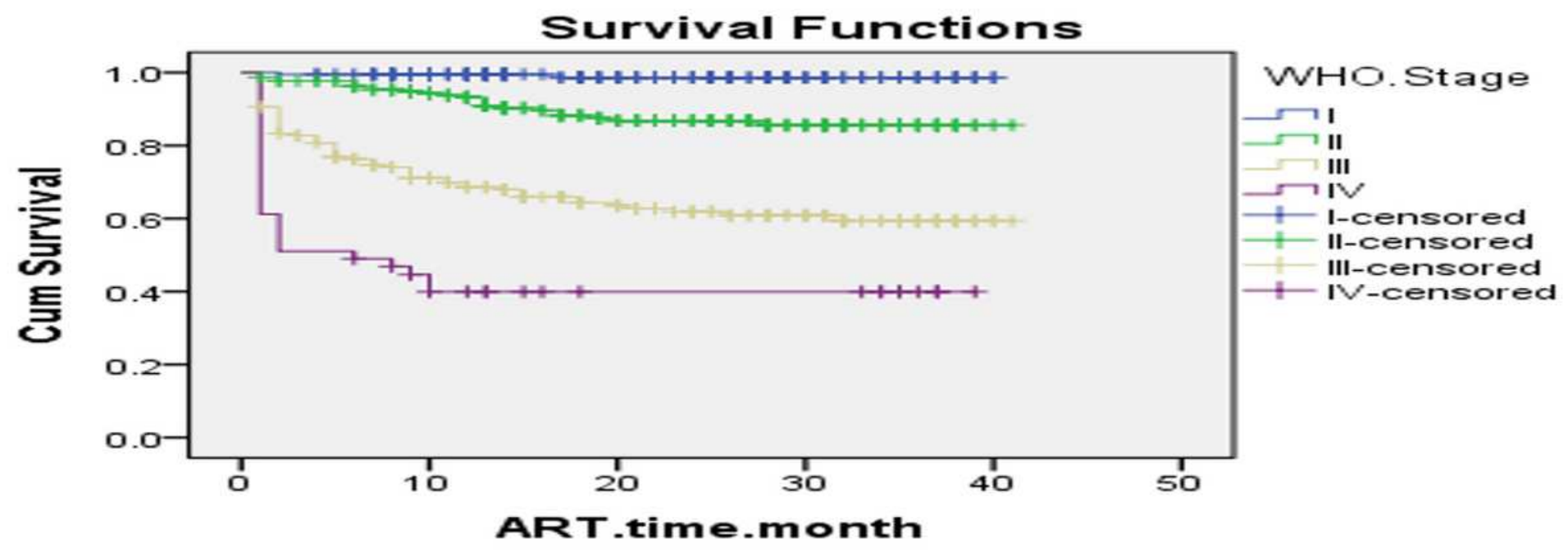

Figure 2

The plot of Kaplan Meier survival estimate for WHO clinical stage 


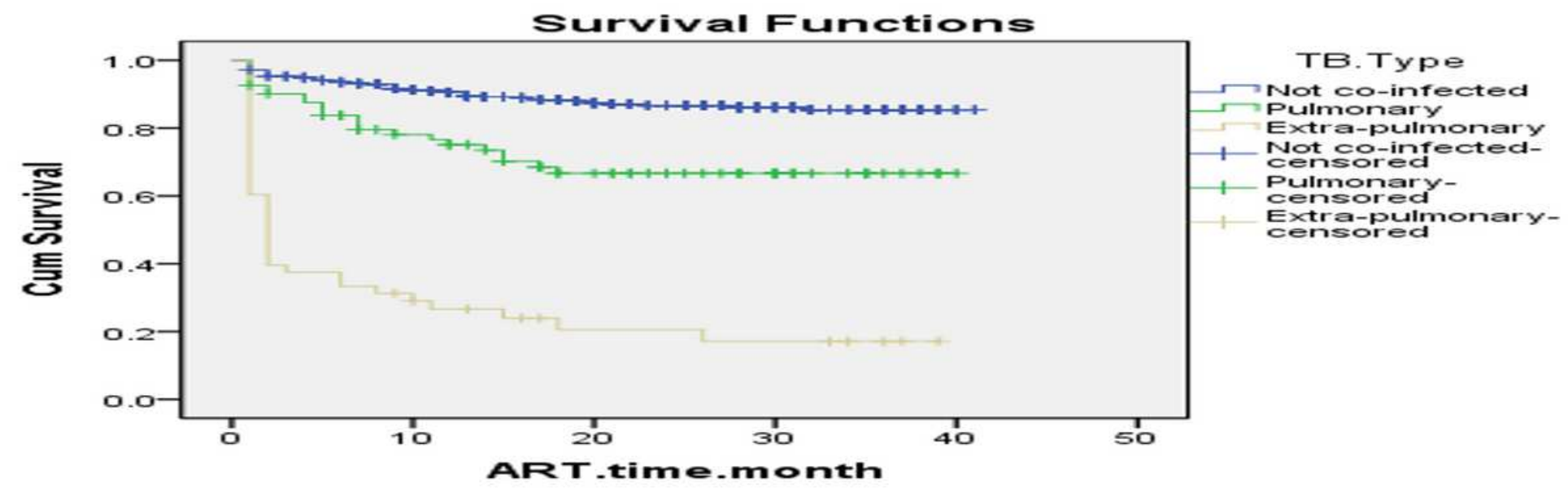

Figure 3

The plot of Kaplan Meier survival estimate for TB type 